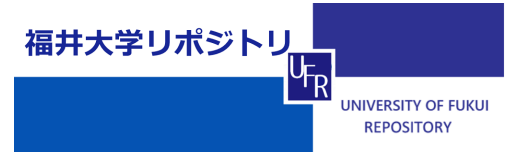

Two- di mensi onal el ect ro- opt i c sampl ing of ter ahert $z$ radi at i on usi ng hi gh- speed compl ement ary met al - oxi de semi conduct or camer a conbi ned wi th arrayed pol arizer

\begin{tabular}{|c|c|}
\hline 著者 & $\begin{array}{l}\text { K TAHARA Hi deaki, TAN Nasahi ko, HANGYO } \\
\text { Nasanor i }\end{array}$ \\
\hline $\begin{array}{l}\text { j our nal or } \\
\text { publ i cat i on title }\end{array}$ & Appl i ed physics I etters \\
\hline vol une & 94 \\
\hline page range & 091119-1-091119-3 \\
\hline year & $2009-03$ \\
\hline URL & ht t p: //hdl . handl e. net /10098/2916 \\
\hline
\end{tabular}

doi: $10.1063 / 1.3095668$ 


\title{
Two-dimensional electro-optic sampling of terahertz radiation using high-speed complementary metal-oxide semiconductor camera combined with arrayed polarizer
}

\author{
Hideaki Kitahara, ${ }^{a '}$ Masahiko Tani, and Masanori Hangyo \\ Institute of Laser Engineering, Osaka University. 2-6 Yamadaoka. Suita, Osaka 565.0871. Japan \\ (Received 17 December 2008: accepted 7 February 2009: published online 6 March 2009) \\ We developed a terahertz imaging system based on two-dimensional electro-optic (EO) sampling \\ using a high speed complementary metal-oxide semiconductor (C.MOS) camera and an arrayed \\ polarizer matched to the camera. By operating the CMOS camera in a normalized differential mode \\ on a single-shot basis, two-dimensional EO sampling is demonstrated. 02009 American Institute of \\ Physics. [DOI: 10.106.3/1.3095668]
}

Terahertz imaging is attracting much attention by virtue of its wide range of potential applications. ${ }^{\text {t- }}$ In 1995. Hu and Nuss ${ }^{5}$ demonstrated the first terahertz imaging based on a system with a paired photoconductive terahertz emitter and detector and with raster scanning of the sample in the focal plane of a terahertz radiation beam. In 1996. Wu et al. ${ }^{6}$ demonstrated the two-dimensional electro-optic (2D-EO) sampling of terahertz radiation using an EO crystal plate and a charge coupled device camera, which reduced the image acquisition time considerably.

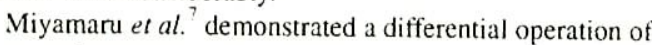
the complementary metal-oxide semiconductor (CMIOS) camera (dynamic subtraction) ${ }^{\S}$ synchronized with pump laser pulses at I kHz for terahertz imaging. The CMOS camera system can create differential images by subtracting the background image from the terahertz field image including the background. These images are alternately generated by chopping the exciting laser beam with an optical chopper. In addition to the dynamic subtraction, each image frame was normalized by the corresponding laser intensity. As a result, a signal-to-noise ratio (SNR) of around 680 was achieved. ?

To maximize the sensitivity, the "nearly zero phase-bias" detection ${ }^{3}$ is usually used in the 2D-EO sampling system. In this case, the response of the EO crystal to the terahertz electric field is not linear. Therefore. observed time-domain waveforms are distorted.

The terahertz beam needs to be defocused to illuminate the whole sample area in the 2D-EO sampling. Because of the low intensity of terahertz radiation, the SNR or the image quality is signiticantly degraded compared to the quality of raster-scanned images. The large intensity fluctuation in the amplified low repetition rate laser beam further reduces the S.NR of terahertz images.

Balanced detection with a $\pi / 2$ phase bias is the best way to solve such problems. ${ }^{9}$ Generally. a pair of optical detectors is used in the balanced detection method. To try to realize balanced detection in 2D-EO sampling. two matched cameras are needed. In addition, the two EO images on the two cameras need to completely coincide in position.

\footnotetext{
Present address: Science and Technology Center for toms. Motecules. and lons Control. Plasma Physics Laboratory. Osaka Lntversity. $2 \cdot 1$ Yamadaoka. Suita, Osaka 565-0871. Japan. Electronic maı kıtahara@ppl eng osaka-u uc.jp.
}

In this paper we demonstrate a balanced 2D-EO sampling using a single camera and an arrayed polarizer. The arrayed polarizer is used to detect the vertical and horizontal polarization components of the circularly polarized optical sampling beam with the laterally sequenced image pixels of the CMOS camera. An image pixel of the camera detects one polarization component and the adjacent pixel detects the orthogonal polarization component through the arrayed polarizer. The pair of signals detected with the adjacent image pixels is used to produce the normalized and balanced EO signal.

Figure I shows the block diagram of the 2D-EO imaging system. We used an amplified femtosecond laser operated at $1 \mathrm{kHz}(\lambda \sim 800 \mathrm{~nm} . \delta t \sim 150$ fs) as the pump source. Terahertz radiation was generated by pumping a ( 110$)$-cut $\mathrm{ZnTe}$ crystal plate ( $1.5 \mathrm{~mm}$ thick). The terahertz beam was focused with a polyethylene lens to the detector ZnTe crystal plate ( $2.5 \mathrm{~mm}$ thick). The EO-sampled image size was matched to the camera's imaging sensor area size. The probe laser beam was combined collinearly with the terahertz beam by a highresistivity silicon beam splitter. The average pump powers on the emitter and probe beam power on the detector were $\sim 200 \mathrm{~mW}$ and $<1 \mu \mathrm{W}$, respectively. The probe beam was $\pi / 2$ phase biased with a variable wave plate in order to make a circularly polarized probe beam in the absence of the terahertz signal to perform the balanced 2D-EO sampling [see Figs. I and 2(a)]. The arrayed polarizer (MPQ-800S, Photonic Lattice Inc.) was placed in front of the CMOS camera (Model C820I. Hamamatsu Photonics K. K.). The arrayed polarizer was fabricated based on the photonic crystal technology. It has a striped pattern with an interval of $40 \mu \mathrm{m}$,

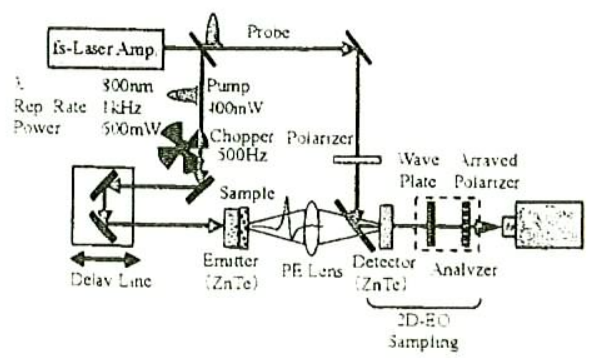

FIG I Color online! Schematte of the 2D-EO imaging system with batunced Jetection 


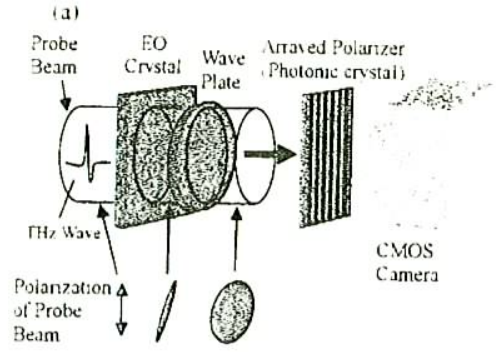

(b)

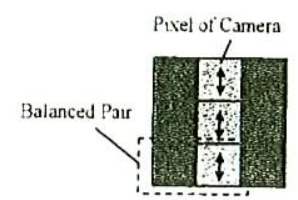

FIG. 2. (Color online) (a) Schematic of the 2D.EO sampling scheme with baianced detection. The polarization of the probe beam has an elliptic shape in front of the amayed polanzer. (b) A part of the arrangement of the polar ization on the CMOS camera. Arrows show the polarization direction of the probe beam.

equal to the pixel interval of the CMOS camera with $128 \times 128$ image pixels on the sensor area of 5.12 $\times 5.12 \mathrm{~mm}^{2}$. The neighboring stripes have polarization axes orthogonal to each other. The arrayed polarizer transmits the vertical and horizontal components of the probe beam onto the camera so that the pixels in an arrayed direction on the camera give rise to the differential pairs of the EO signal [see Fig. 2(b)].

The terahertz image was obtained by the C.MOS camera for each laser shot triggered by a $1 \mathrm{kHz}$ synchronous output from the laser driver. The linearly polarized components of the probe laser with the horizontal polarization transmitted through the arrayed polarizer were detected by every two lines of pixels on the camera. The orthogonally polarized components of the probe laser with the vertical polarization were detected by the other set of lines of pixels on the camera. The balanced and normalized 2D-EO signals were obtained by the following equation:

$$
\operatorname{Img}_{\text {balance }}[i, 2 j]=\frac{\operatorname{Img}[i, 2 j-1]-\operatorname{Img}[i, 2 j]}{\operatorname{Img}[i, 2 j-1]+\operatorname{Img}[i, 2 j]} \text {. }
$$

where $\operatorname{Img}[i, j]$ represents the signal for the $i$ th row and $j$ th column of the image pixels. Here we assume that the pixels of the $(2 j-1)$ th row detect the horizontally polarized components and those of the $2 j$ th row detect the vertically polarized components. Img balance $[i, 2 j]$ is expected to be linear to the terahertz field and free from the laser intensity fluctuation.

The image in Fig. 3 is a background noise image without terahertz wave signals, taken at a rate of $10 \mathrm{frame} / \mathrm{s}$ (averaged for 100 balanced images). When the probe beam is blocked, the image is blacked out. According to Eq. (1), the background noise should be completely suppressed by the differential detection in the ideal case. However, a large noise is observed in the background image as seen in Fig. 3. In real-time image observation. the numerical values of all the pixels were fluctuating randomly. Therefore, the image

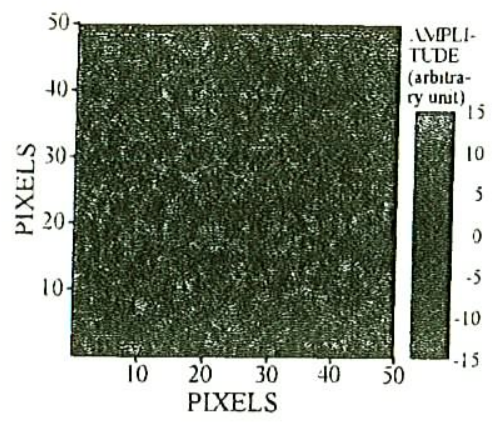

FIG. 3. (Color online) Distnbution of the noise in the cross section of the balanced image without terahertz electric fields. The image represents the intensity fluctuation in the cross section of the probe beam. Ideally the Image should be shown in black.

did not show any fixed pattem in time. If the nonuniformity of the ZnTe crystal influenced, then the element in the arrayed polarizer, or any optical components, should reveal a fixed pattern in the real-time image. This contradicts the experimental result. When hot air was blown into the path of the probe beam by a hair dryer. the terahertz images were strongly distorted. Therefore. we guess that the naturally fluctuating air density in the laboratory room caused the nonuniformity of the intensity distribution of the probe beam, resulting in the background noise observed in Fig. 3.

In the above detection scheme. the adjacent positions in the cross section of the probe beam corresponding to the adjacent pixels of the CMOS camera are used to balance the signal, assuming that the adjacent positions have nearly the same intensity for the probe beam and nearly the same terahertz electric field. However, the actual probe intensity is not uniform and the neighboring pixels give rise to significantly different signal values. which fluctuate in time. This is why the background noise cannot be completely suppressed by using the balanced detection described above. Therefore, we tried to reduce this noise by performing time integration and dynamic subtraction. ${ }^{7.3}$

Dynamic subtraction is used to suppress the noise. the variation of which is slower than the sampling time. A terahertz wave was generated for every two laser pulses using an optical chopper. ${ }^{3 . x}$ Thus, the series of images contain the terahertz signal altemately. The image generated by the dynamic subtraction for the balanced 2D-EO system is then given by the following equation:

$$
\text { Img batance +ditf }=\sum_{k=1}^{N}\left(\operatorname{Img}_{\text {balance }}^{2 k}-\operatorname{Img}_{-}^{2 k-t} \text { balance }\right) .
$$

In Eq. (2), $N$ is the number of the pair of images with and without the terahertz signal in the averaging procedure. In the even event (suffix $2 k$ ) Img baiance contains the terahertz signal. whereas in the odd event (suffix 2k-1) Img_balance does not contain the terahertz signal. To measure images at various time delays (or 2D terahertz waveforms). the optical delay line was moved step by step and the differential images were accumulated for a tixed time duration at each step. All this processing, including the normalization. the dynamic subtraction, and the time integration, were performed on an arithmetic card implemented by a control computer. 


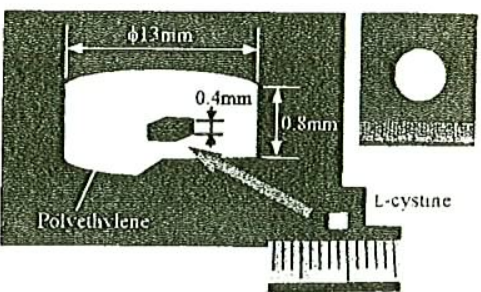

FIG. 4. (Color online) Schematics of the outline and structure of the sample. The sampie is a circuiar pellet. The outer shell of the sample is made of polyethylene. A piece of L-cystine is embedded in the center of the sample.

The test sample for imaging was a polyethylene pellet in which a piece of L-cystine plate was embedded. The structure of the sample is shown in Fig. 4. The L-cystine plate measured $2 \times 2 \times 0.4 \mathrm{~mm}^{3}$ and the polyethylene pellet was $\phi 13$ and $0.8 \mathrm{~mm}$ thick. Because polyethylene and L-cystine have different refractive indices, the two regions should be distinguishable from the time delay of the peaks in the terahertz waveforms.

In the experiment, we observe the terahertz image of the $\mathrm{L}$-cystine in the polyethylene pellet at a fixed time delay where the terahertz peak amplitude in the L-cystine region was almost maximum. The image of the normalized balanced signal of the test sample shown in Fig. 5 is produced from 100 frame integration by using Eq. (2). The real-time

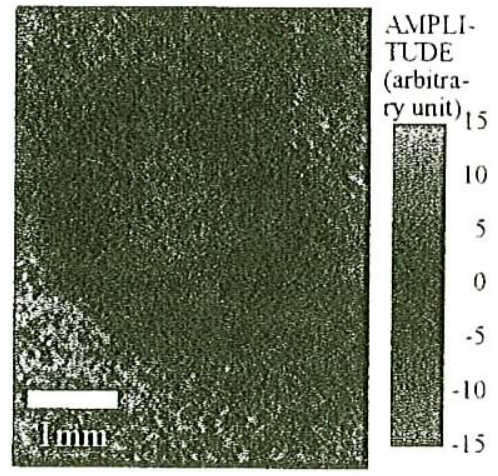

FIG. 5. (Color online) (mage of L-cystine embedded in the polvethviene pellet. The acquired image region is $5 \times+\mathrm{mm}^{2}$ images ${ }^{(1)}$ are also obtained from the successive acquisition of the differential, normalized images. The L-cystine plate is clearly observed in the real-cime image (10 frames/s). However. the L-cystine appears to be round owing to the low spatial resolution of the image due to the diffraction effect for the terahertz radiation. whose wavelengths ranged from 0.15 to $3 \mathrm{~mm}$. The spatial resolution is evaluated to be approximately $1 \mathrm{~mm}$. In the terahertz image, the terahertz EO signal in the four corners of the imaged area is almost zero owing to the small diameter of the terahertz beam. Therefore. the available imaging area was restricted within a circle of 5 $\mathrm{mm}$ diameter. In the evaluation of the SNR, the noise is defined as the fluctuation of the EO signal in a particular pixel for different frames when a series of images were acquired at the fixed time delay. An SNR around 10 is obtained for the image around the center of the terahertz beam with an accumulation time of $0.1 \mathrm{~s}$ ( 100 shots on average).

In conclusion, we demonstrated a balanced 2D.EO sampling of terahertz radiation with an arrayed polarizer and a high speed CMOS camera operated in a dynamic subtraction mode. A reasonable image SNR around 10 is achieved for a rate of $10 \mathrm{frame} / \mathrm{s}$.

This work has been supported in part by the Terahertz Optics Project for Medical Application led by J. Nishizawa, organized by the Ministry of Education, Culture, Sports, Science, and Technology of Japan. The authors thank Dr. Shojiro Kawakami tor his advice in preparing the arrayed polarizer.

D. M. Mitteman. S. Hunsche. L. Botvin. and M. C. Nuss. Opt. L.ell. 22 904 (1997)

'B Ferguson. S Wang, D Gray. D Abbot, and X C. Zhang, Opt L.ett. 27. 1312 12002)

K. J. Siebert, H. Quast, R. Leonhardt. T. Loffler. M. Thomson, T. Bauwe.

H. G. Roskos. and S. Crasch. Appi. Phis. Lett. 80. 3003 (2002).

K. Kawase. Y Ogawa, and Y. Watanabe. Opt. Express 11. 2549 (2003).

B. B. Hu and M. C. Nuss. Opt L.ent. 20. 17!6 \$1995

'Q. Wu, F G. Sun. Q Chen. and X.C. Zhang. Appt. Plus L.en!. 69.1026 (1996)

F. Miyamaru. T. Yonera. M. Tan. and M. Hangyo, jpm. J. Appl Pli:, Part 2 43. L $489(2004)$

'Z. Jiang. X. G. Xu, and X.C. Zhang. Appt. Opt. 39. 2982 120001

'Z. Jiang. F G. Sun. Q. Chen. and X.C. Zhang. Appi P!iv 1.2it 74. 1191 (! 9991 .

See EPAPS Document No. E-APPLAB-94-1076910 tor the real-ume images. For tnore information on EPAPS, iee hit //www ap.org/ pubservs/epaps.huml 